\title{
A MODIFIED CRANK-NICOLSON TECHNIQUE WITH NON-EQUIDISTANT SPACE STEPS
}

\author{
G. EIGENBERGER ${ }^{\dagger}$
}

Institut für Systemdynamik und Regelungstechnik, (Direktor: Prof. Dr.-Ing. E. D. Gilles), Universitāt Stuttgart, W. Germany

and

\section{J. B. BUTT}

Department of Chemical Engineering, Northwestern University, Evanstone, IL 60201, U.S.A.

\author{
(Receired 24 November 1975; accepted 16 February 1976)
}

\begin{abstract}
A finite difference method with non-equidistant space steps, based upon the Crank-Nicolson technque is presented. Its prime feature is the automatic positioning of axial grid points at required positions, thus reducing considerably the total number of grid points and hence the amount of computer time.

The method is demonstrated for a number of examples of tubular reactor calculations. It proves to be well suited for the solution of all kinds of diffusion type models, especially if steep gradients or moving profiles occur, and can be used even on moderate size process computers.
\end{abstract}

Since many problems in chemical reaction engineering ultimately require the solution of sets of nonlinear partial differential equations, there is a continuing need for the development of numerical methods which can accomplish the task with computation time and storage requirements as small as possible. Among the vast variety of methods which have been proposed, two stand out as having been used extensively and with good success: the classical Crank-Nicolson technique[1] combined with quasilinearization [2], and the method of orthogonal collocation $[3,4]$. However, in reaction and reactor modelling for highly exothermic reactions in which very steep concentration and temperature gradients are commonly encountered, both of these methods have significant drawbacks. The main disadvantage of the CrankNicolson technique is the requirement of a large number of equidistant space steps (usually 200 or more), resulting in a large amount of computer time. Drawbacks of the method of orthogonal collocation result from the fact that the spatial grid points are determined by the zeros of the orthogonal polynomial which, of course, have nothing to do with the solution profile. This is particularly detrimental in case of unsteady or creeping profiles, where the grid points should be concentrated in the (moving!) region of maximum reaction rates. An increase in the number of grid points (i.e. in the degree of the orthogonal polynomial) again results in a better approximation but at the expense of a considerably increased amount of computer time, since a set of fully occupied matrices has to be inverted instead of a set of tridiagonal matrices as in the case of the Crank-Nicolson method. In reaction engineering this is apparently the reason why the method of orthogonal collocation has mostly been applied to single pellet diffusion-reaction problems or to the approximation of radial profiles in tubular reactor calculations-both cases with relatively smooth profiles.

†New address: BASF AG, Abt. TEI, D6700 Ludwigshafen.
Figure 1 shows typical concentration and temperature profiles of an exothermic reaction in a nonadiabatic tubular reactor. It is obvious that in the range of the maximum temperature, the grid points have to be concentrated very close together to obtain accurate approximations of the axial derivatives (and of the source terms), whereas in the fore- and aft section large space increments could be allowed. Thus the density of axial grid points required for accurate approximation varies with position within the reactor, and a considerable reduction from the number required in an equidistant spacing scheme should be possible.

In numerical analysis finite difference methods with non-equidistant spacing can be found under the heading "mesh refinement" (see [14]-[18]). Analysis is focused upon the problem of stability if two or three regions with different (but in each region equidistant) space steps are used. However, little attention has been devoted to the problem of optimal positioning of grid points. An early treatize can be found in [10].

Probably the most advanced method to date has been developed by Lübeck $[5,6]$ and demonstrated in a number of examples $[7,8]$. It is based upon the solution of an

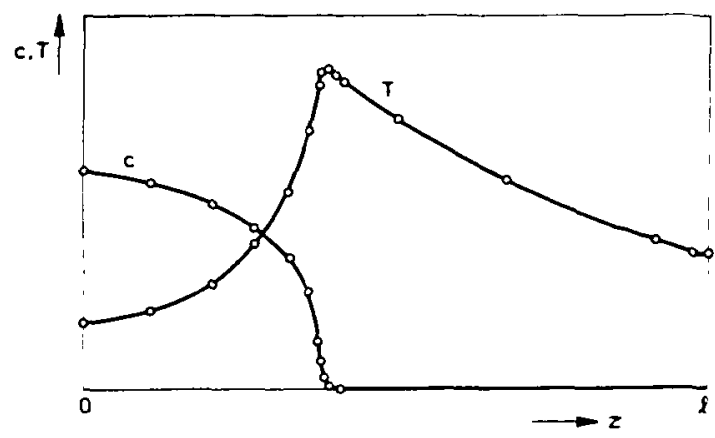

Fig. 1. Concentration and temperature profiles in a tubular reactor and approximate position of required grid points (circles) for numerical solution. 
integral form of the balance equations and uses a fixed number of spatial grid points (usually 10-30) which are repositioned during each time step to represent optimally the shape of the solution profiles. The main advantages of Lübeck's method are its global stability and small computer time requirements, but it requires large storage capacity and programming effort.

Recently Carey and Finlayson [9] published a method of orthogonal collocation on finite elements in which the space domain was split into a number of (non-equidistant) elements, each of which was described by a low order collocation polynomial. The amount of the residuals could be used as guideline to position the elements optimally. Compared to usual orthogonal collocation the main advantages are optimal position of elements (grid points) and a resulting block-diagonal matrix instead of a fully occupied matrix. However, the method was tested only for the simple example of one steady state balance equation for a single pellet.

In the following we present a finite difference method with non-equidistant space steps, based on the CrankNicolson technique which has proven to be well suited for solving a variety of reaction engineering problems involving diffusion-type models even on a moderate size process computer.

\section{PART 1-THE ALGORTHM}

\section{General concept}

One way to derive a finite difference approximation for the spatial differential quotient is to assume that the profiles of the dependent variables can be piecewise approximated by second order parabolas. This is shown in Fig. 2 where it is assumed that the differential equation is exactly fulfilled in the circled points. If the solution $y(z)$ is represented piecewise by the depicted parabolas, certain errors are introduced. An approximation for the maximum error in Fig. 2 between $z_{L-1}$ and $z_{L}$ is the difference $E$ between the parabolas at $z^{\prime}=$ $\left(z_{L-1}+z_{L}\right) / 2$. The knowledge of this error provides an easy means for an automatic space step control: If the error is above a specified minimum accuracy level, an additional grid point has to be inserted at $z^{\prime}$. If two subsequent errors are below another specified maximum

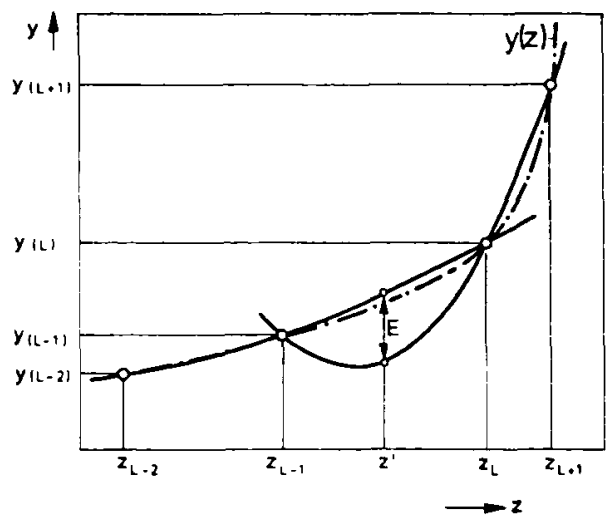

Fig. 2. Approximation of a solution profile $y(z)$ (dotted line) by second order parabolas (solid lines). accuracy level, the grid point in between can be omitted in subsequent calculations.

Thus the spatial accuracy will always be kept within a prespecified range and the grid points are positioned in an optimal way (contrary to methods with equidistant grid points, where one is never sure whether the accuracy in zones of steep gradients or maxima is sufficient).

The modified Crank-Nicolson technique will be developed here for the case of a general diffusion-type model for tubular reactors which consists of partial differential equations of the form

$$
\frac{\partial y}{\partial t}=D_{\mathrm{eff}} \frac{\partial^{2} y}{\partial z^{2}}+v \frac{\partial y}{\partial z}+S(y, z)
$$

with boundary conditions

$$
\left.\frac{\partial y}{\partial z}\right|_{z=0}=\frac{v}{D_{\text {eff }}}\left(y_{(z=0)}-y_{0}\right) ;\left.\quad \frac{\partial y}{\partial z}\right|_{z=1}=0 .
$$

Finite difference representation of the spatial differential quotient

During the calculation $y$ will be evaluated at certain grid points $z_{L}, L=1,2, \ldots, L M$, as shown in Fig. 3. It turns out that it is useful for the derivation of the spatial difference approximation in a nonequidistant grid to distinguish between the grid points and the elements to which they belong. In Fig. 3 it can be seen that the grid point $z_{L}$ lies almost at the boundary of its (shaded) element which is defined to extend from $\left(z_{L-1}+z_{L}\right) / 2$ to $\left(z_{L+1}+z_{L}\right) / 2$. A finite difference approximation which is evaluated at $z=z_{L}$ (as well as the source term $S\left(y_{L}, z_{L}\right)$ ) therefore only imperfectly represents the mean value in the element and it seems more reasonable to evaluate the difference approximation for the middle of the element at

$$
z=\bar{z}_{L}=\frac{\left(z_{L-1}+2 z_{L}+z_{L+1}\right)}{4}
$$

instead.

The spatial differential quotient for each element can be approximated in two different ways, which, in case of nonequidistant space steps, lead to slightly different results. One way is to write balance equations for each element which has advantages in certain instances (see eqn 9). The second way, which will be used here, is to place a second order parabola through the grid points

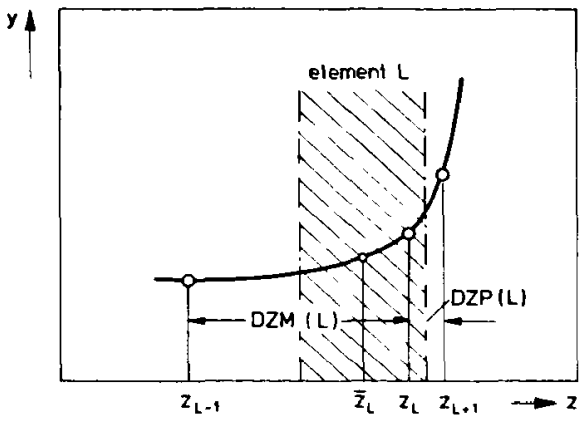

Fig. 3. Grid points and elements in a non-equidistant grid. 


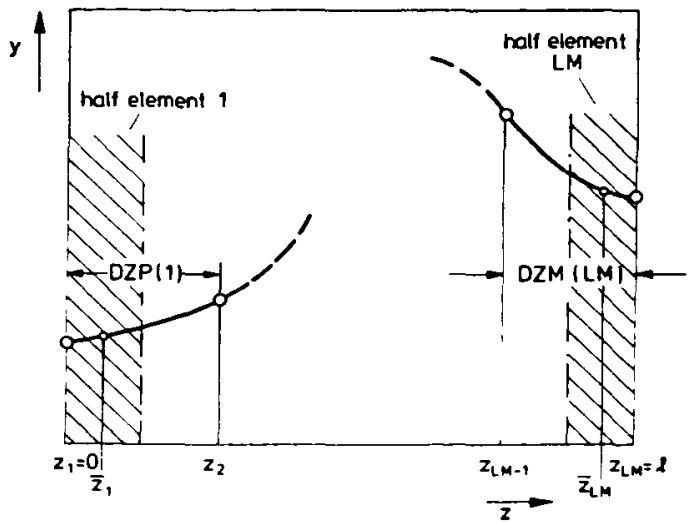

Fig. 4. Half elements at the beginning and at the end of the space domain.

$(L-1), L,(L+1)$ and to evaluate its derivatives at $z=\bar{z}_{L}$. Thus

$$
\begin{aligned}
D_{\mathrm{eq}} \frac{\mathrm{d}^{2} y}{\mathrm{~d} z^{2}}-\left.v \frac{\mathrm{d} y}{\mathrm{~d} z}\right|_{z=z_{L}}= & C(L, 1) y(L-1)+C(L, 2) y(L) \\
& +C(L, 3) y(L+1)
\end{aligned}
$$

where

$$
\begin{aligned}
& C(L, 1)=\frac{2 D_{\mathrm{ef}}+0.5 v(D Z M(L)+D Z P(L))}{D Z M(L)(D Z M(L)-D Z P(L))} \\
& C(L, 2)=-\frac{4 D_{\mathrm{eff}}+v(D Z P(L)-D Z M(L))}{2 D Z M(L) D Z P(L)}
\end{aligned}
$$$$
\text { for } L=2 \text { to } L M-1 \dagger
$$

$C(L, 3)=-(C(L, 1)+C(L, 2))$

The approximation is the same for all elements except for the first and the last one. As can be seen in Fig. 4, only half elements are left at the beginning and the end of the reactor and the difference approximation has to be evaluated in their middle, i.e. at $\bar{z}_{1}=D Z P(1) / 4$ and $\bar{z}_{L M}=z_{L M}-D Z M(L M) / 4$. Two values of $y$ at the grid points and the respective boundary condition will be used to calculate the parabolas and to determine the differential quotient at $\bar{z}_{1}$ and $\bar{z}_{L M}$. Thus

$D_{\mathrm{eff}} \frac{\mathrm{d}^{2} y}{\mathrm{~d} z^{2}}-\left.v \frac{\mathrm{d} y}{\mathrm{~d} z}\right|_{:=\mathrm{s}_{1}}=D E(1)+C(1,2) y(1)+C(1,3) y(2)$

where

$$
\begin{aligned}
& C(1,2)=-\frac{2 D_{\mathrm{en}}}{D Z P(1)^{2}}-\frac{3}{2} \frac{v}{D Z P(1)}-\frac{v^{2}}{2 D_{\mathrm{ef}}} \\
& C(1,3)=\frac{2 D_{\mathrm{eft}}}{D Z P(1)^{2}}-\frac{v}{2 D Z P(1)} \\
& D E(1)=-y_{0}(C(1,2)+C(1,3))
\end{aligned}
$$

and

$$
\begin{aligned}
D_{\mathrm{eff}} \frac{\mathrm{d}^{2} y}{\mathrm{~d} z^{2}}-\left.v \frac{\mathrm{d} y}{\mathrm{~d} z}\right|_{z=z_{L M}}=C(L M, 1) y(L M-1) \\
-C(L M, 2) y(L M)
\end{aligned}
$$

†Notation is chosen close to that used in programming languages. where

$$
C(L M, 1)=-C(L M, 2)=\frac{2 D_{\mathrm{ef}}}{D Z M(L M)^{2}}+\frac{v}{2 D Z M(L M)}
$$

The difference approximation at the entrance based on the parabolic representation (eqns 5,6 ) is limited to diffusivities $D_{\text {ef }}>0$. For small values of $D_{\text {eff }}$ a satisfactory approximation can be obtained using the first mentioned method above, i.e. a balance equation for the first element (reactor cross section A):

\section{$A \frac{D Z P(1)}{2} \frac{\mathrm{dy}(1)}{\mathrm{d} t}=$}

$$
\underbrace{A v\left\{y_{0}-\frac{y(1)+y(2)}{2}\right\}}_{\text {convection }}+\underbrace{D_{\mathrm{ef}} A \frac{y(2)-y(1)}{D Z P(1)}}_{\text {diff usion }}+\underbrace{A \frac{D Z P(1)}{2} \bar{S}(1)}_{\text {source term }}
$$

$$
\begin{aligned}
& \frac{\mathrm{d} y(1)}{\mathrm{d} t}=\underbrace{\frac{2 v}{D Z P(1)}}_{D E(1)} y_{0}-\underbrace{-\left(\frac{2 D_{\mathrm{eA}}}{D Z P(1)^{2}}+\frac{v}{D Z P(1)}\right)}_{C(1,2)} y(1) \\
& \underbrace{+\left(\frac{2 D_{\mathrm{ef}}}{D Z P(1)^{2}}-\frac{v}{D Z P(1)}\right)}_{C(1,3)} y(2)+\bar{S}(1) \text {. }
\end{aligned}
$$

The original diffusion equation and boundary conditions are now reduced to a set of $L M$ ordinary differential equations in time which have the general form

$$
\begin{aligned}
\frac{\mathrm{d} y(L)}{\mathrm{d} t}= & C(L, 1) y(L-1)+C(L, 2) y(L) \\
& +C(L, 3) y(L+1) \\
& +D E(L)+\bar{S}(L)
\end{aligned}
$$

where

$$
D E(L)=0 \text { for } L \neq 1
$$

\section{Complete difference form of model equations}

Following the Crank-Nicolson technique, set (10) of ordinary differential equations is solved using the trapezoidal rule:

$$
\frac{\mathrm{d} y}{\mathrm{~d} t}=f(y) \rightarrow y^{k+1}=y^{k}+\frac{\Delta t}{2}\left(f_{(y)}^{k+1}+f_{(y)}^{k}\right)
$$

where $k$ is the time index. Since the nonlinear source term $S(y, z)$ is part of the right hand side $f(y)$, the above equation has to be solved iteratively. In order to obtain fast convergence of the iterations, the quasilinearization technique can be applied[2]:

$$
f_{p}^{k+1}=f_{p-1}^{k+1}+\frac{\partial f^{k+1}}{\partial y_{p-1}}\left\{y_{p}^{k+1}-y_{p-1}^{k+1}\right\}
$$

where $p$ is the iteration index. 
Thus the trapezoidal rule with quasilinearization gives

$$
y_{p}^{k+1}\left\{1-\frac{\Delta t}{2}{\frac{\partial f^{k+1}}{\partial y_{p}-1}}^{k}=y^{k}+\frac{\Delta t}{2}\left\{f_{p-1}^{k+1}-\frac{\partial f^{k+1}}{\partial y_{p-1}} y_{p-1}^{k+1}+f^{k}\right\} .\right.
$$

If this result is applied to the set of differential equations (10), a tridiagonal system of linear equation for the unknowns $y_{p}{ }^{k+1}(L), L=1$ to $L M$, can be obtained:
These nonlinear equations can be linearized by the quasi-linearization technique and the system of tridiagonal linear eqns (12) again results except that the values for $D A(L)$ and $A L(L, 2)$ are now given by:

$$
\begin{aligned}
D A(L) & =D E(L) \frac{\Delta t}{2} \\
A L(L, 2) & =A L(L, 4)-1+\frac{\Delta t}{2} \frac{\partial S^{k+1}}{\partial y_{p-1}} .
\end{aligned}
$$

$$
\begin{aligned}
& A L(1,2) y_{p}{ }^{k+1}(1)+A L(1,3) y_{p}{ }^{k+1}(2) \\
& =D D(1)
\end{aligned}
$$

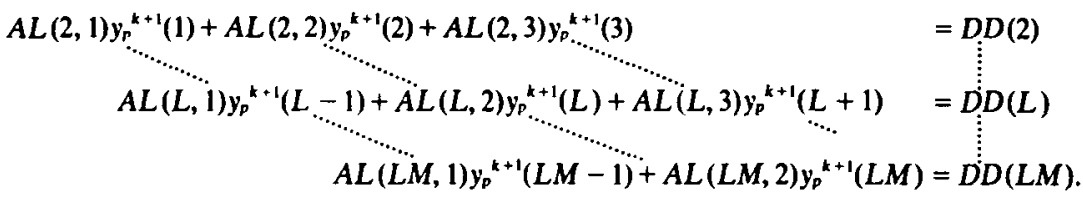

Where

$$
\begin{aligned}
& A L(L, 1)=\frac{\Delta t}{2} C(L, 1) ; A L(L, 3)=\frac{\Delta t}{2} C(L, 3) \\
& A L(L, 4)=\frac{\Delta t}{2} C(L, 2)+1 ; \\
& A L(L, 2)=A L(L, 4)-2+\frac{\Delta t}{2} \frac{\partial S^{k+1}}{\partial y_{p-1}} \\
& D D(L)=-D A(L)-\frac{\Delta t}{2}\left\{\bar{S}_{p-1}^{k+1}(L)-\frac{\partial S^{k+1}}{\partial y_{p-1}} y_{p-1}^{k+1}(L)\right\} \\
& D A(L)=A L(L, 1) y^{k}(L-1)+A L(L, 4) y^{k}(L) \\
& +
\end{aligned}
$$

This result is basically the same as in the Crank-Nicolson method with equidistant space steps, except that the coefficients $A L(L, i)$ change with $L$ and have to be recalculated if the spatial grid changes.

$A L(L, 1), A L(L, 3) A L(L, 4)$ and $D A(L)$ stay constant during the iteration cycle, only $A L(L, 2)$ and $D D(L)$, i.e. coefficients containing the source term and its derivatives at time $(k+1)$ have to be recalculated in each iteration step.

Usually, there is more than one balance equation, in which case for every balance equation such a tridiagonal system can be formulated. The systems are coupled by their source terms, but can be solved independently at each iteration step.

\section{Balance equations in (quasi) steady state}

Often some of the balance equations can be considered to be in quasi steady state. Then eqn (10), multiplied by $\Delta t / 2$ reads

$$
\begin{gathered}
\frac{\Delta t}{2} C(L, 1) y(L-1)+\frac{\Delta t}{2} C(L, 2) y(L)+\frac{\Delta t}{2} C(L, 3) y(L+1) \\
=-\{D E(L)+\bar{S}(L)\} \frac{\Delta t}{2} \text { for } L=1 \text { to } L M .
\end{gathered}
$$

Thus dynamic and steady state calculations can be performed with the same algorithm.

\section{Adjustment of spatial grid points}

After the iterations for one time step have converged, the result is checked for spatial accuracy. As outlined at the beginning, the decision whether an additional grid point has to be inserted or an existing grid point can be omitted depends upon the error $E$ of the approximation. This error is defined as the difference between two adjacent parabola-approximations, half-way between the grid points (Fig. 5):

$$
E(L)=Y H M(L+1)-Y H P(L) .
$$

Thus "half values" $Y H M, Y H P$ have to be calculated for each grid point. The parabolic approximation gives

$$
\begin{aligned}
\operatorname{YHM}(L) & =y\left(\frac{z_{L-1}+z_{L}}{2}\right) \\
& =H M(L, 1) y_{L-1}+H M(L, 2) y_{L}+H M(L, 3) y_{L+1} \\
Y H P(L) & =y\left(\frac{z_{L}+z_{L+1}}{2}\right) \\
& =H P(L, 1) y_{L-1}+H P(L, 2) y_{L}+H P(L, 3) y_{L+1}
\end{aligned}
$$

where $H M$ and $H P$ for $L=2$ to $L M$ are given in Table 1 .

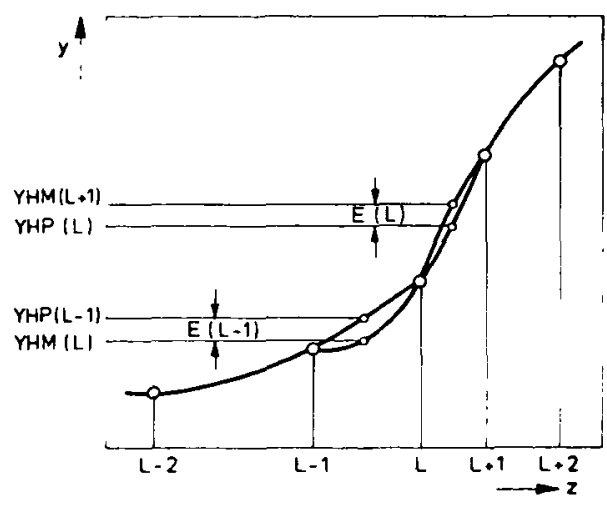

Fig. S. Determination of the error $E$ of the approximation. 
Table 1. Constants of the modified Crank-Nicolson method

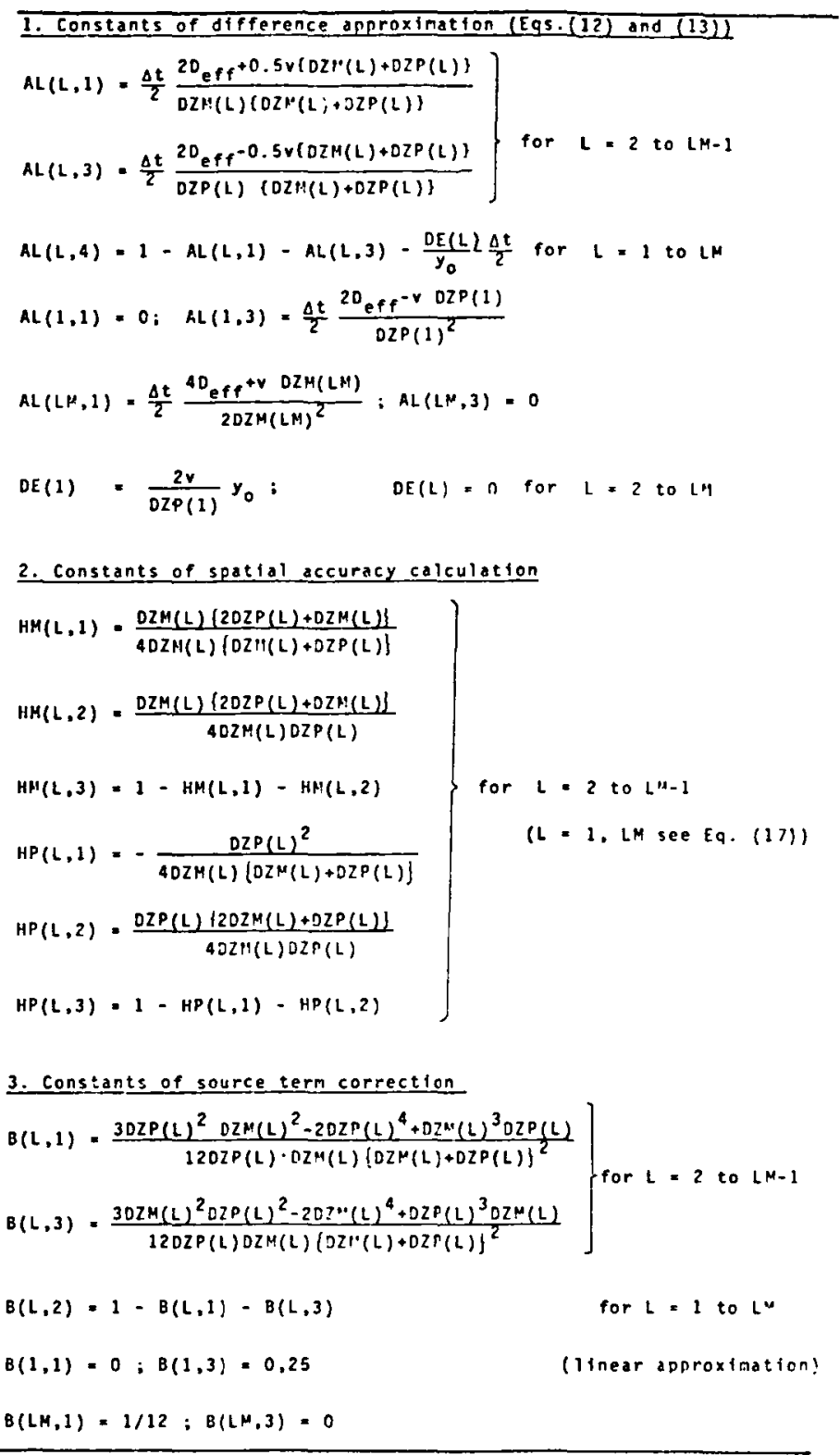

Using the boundary condition at $z=0$ and $z=l$, the half values for the entrance and exit section are

$$
\begin{aligned}
Y H P(1)= & y\left(\frac{z_{1}+z_{2}}{2}\right) \\
= & -\frac{v D Z P(1)}{4 D_{\text {efl }}} y_{0}+\left\{\frac{3}{4}+\frac{v D Z P(1)}{4 D_{\text {eff }}}\right\} y(1) \\
& +\frac{1}{4} y(2) \\
Y H M(L M)= & y\left(\frac{z_{L M-1}+z_{L M}}{2}\right)=\frac{3}{4} y_{L M}+\frac{1}{4} y_{L M-1} .
\end{aligned}
$$

If the relative value of $E(L)$ is larger than a specified limit $\epsilon_{\max }$ a new grid point at $\left(z_{L}+z_{L+1}\right) / 2$ will be inserted. The values of the variables at this point are set to be the mean between $Y H P(L)$ and $Y H M(L+1)$ :

$$
y\left(\frac{z_{L}+z_{L+1}}{2}\right)=\frac{Y H P(L)+Y H M(L+1)}{2} .
$$

If two successive errors are below another specified value $\epsilon_{\max }$ the grid point in the middle will be omitted. To avoid halving and doubling of the same interval in successive time steps, the condition

$$
\epsilon_{\operatorname{man}} \approx 0.1 \epsilon_{\max }
$$

has proved useful. The choice of $\epsilon_{\max }$ and its influence upon the solution will be discussed in the second part of the paper. 


\section{Source term correction}

In Fig. 3 it was shown that in case of nonequidistant space steps it is better to evaluate a mean value for the spatial derivatives and the source term in the interval rather than to use the value at the grid point. (This is not a necessary condition for the method proposed, but it usually increases the accuracy of the approximation.) In the case of the spatial differential quotient this requirement is fulfilled by evaluating the derivatives in the middle of the interval at $z_{L}=\bar{z}_{L}$ (eqn 3). Since the source terms $S\left(y(L), z_{L}\right)$ can be calculated only at the grid points, these values have to be corrected. One possibility consists of calculating the integral mean value for the element, assuming that the variation of the source term within the interval can also be approximated by a parabola. The result is

$$
\begin{aligned}
\bar{S}(L)= & \frac{2}{D Z P(L)+D Z M(L)} \int_{z_{L}-D Z M(L) / 2}^{z_{L}+D Z P(L) / 2} S(y(z), z) \mathrm{d} z \\
= & B(L, 1) S(L-1)+B(L, 2) S(L) \\
& +B(L, 3) S(L+1)
\end{aligned}
$$

where $B(L, i)$ are given in Table 1 .

\section{Sag effect}

Throughout the method second order parabolas have been used for approximation because of their simplicity and because of the fact that second order approximations on short intervals are usually superior to higher order approximations on larger intervals. However, there is one pitfall which is illustrated by Fig. 6 .

Consider the dotted line which shows some function $f(z)$, e.g. temperature or reaction rate in a tubular reactor. The parabolic approximation through $(L-1), L$ and $(L+1)$ represents $f(z)$ badly. This "sag"-effect has two important consequences. The first is the calculation of the integral mean $\bar{S}(L)$ in element $L$ which would be considerably underestimated in the representation of Fig. 6. The second case occurs if a new value at $z_{N}=$ $\left(z_{L}+z_{L-1}\right) / 2$ has to be calculated using eqn (18). In Fig. 6 $Y H M(L)$ is much too low and hence $y\left(z_{N}\right)$ too. The detection of sag effects is easy if they generate a maximum or a minimum as in Fig. 6. If, e.g. the new value $y\left(z_{N}\right)$ exceeds both neighborng values $y(L-1)$ and $y(L)$, a linear interpolation for $y\left(z_{N}\right)$ has to be applied instead.

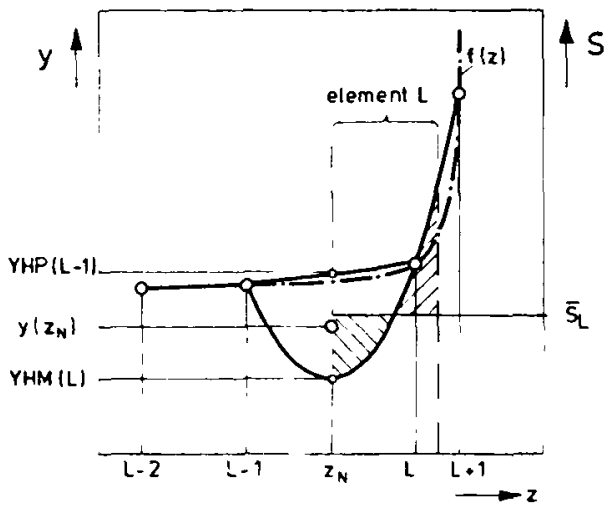

Fig. 6. The occurance of sag effects.

\section{Time step control}

The dynamics of concentration and temperature in chemical reactors often have markedly different time constants, so different time steps should be used depending upon which dynamics are dominant. To reduce computer time, an automatic time step control is advantageous. The following criteria can be used: 1 The maximum variation of one or several variables during one time step; 2 the number of iterations during one time step or 3 a combination of both.

In the examples to be discussed below, the criterion for increase or decrease of the time step $\Delta t$ was the number of iterations required. Four to eight iterations were considered optimal (no change of $\Delta t$ ); fewer iterations permitted an increase in $\Delta t$, more iterations a decrease.

With this time step control the algorithm showed good stability in all examples considered. (It should be mentioned that a successive increase of $\Delta t$ generally tends to destabilize the Crank-Nicolson algorithm. Therefore, a time step increase should be allowed only every fifth or so time step.)

\section{SUMMARY}

The algorithm proposed is summarized in the flow sheet, Fig. 7. The coefficients which have to be calculated at the beginning of the program and each time the space grid is changed are given in Table 1. Compared to the usual Crank-Nicolson technique with equidistant space steps only block (1) and (1) are new in the program. Since the computation time for one stime step is essentially determined by the time required to calculate the nonlinear source term and its derivatives (block (3)) plus the time required to solve the system of tridiagonal equations (block (5), the additional time requirements are negligible. In fact a multiple of computation time can be saved by the reduction of the number $L M$ of axial grid points

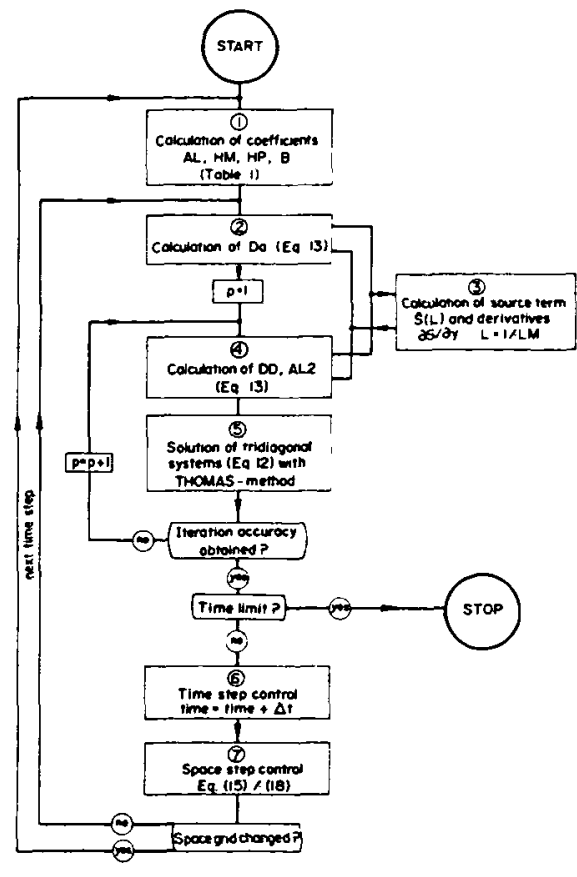

Fig. 7. Program fiow sheet. 
since the amount of computer time is roughly proportional to $L M$. The savings depend upon the problem to be solved and will be discussed in the following examples.

The algorithm has been developed here for diffusionconvection problems but it is obvious that the same technique can also be used to derive a non-equidistant difference scheme for single pellet problems of different geometry. In fact, if the velocity $v$ in the above equations is set to zero, the algorithm can be used for the calculation of single pellet problems with slab geometry.

\section{PART 2-APPLICATIONS}

To show its feasibility, the method proposed is demonstrated for a number of examples. All calculations have been performed in FORTRAN IV on a moderate size 16 bit, double word process computer (EAI Pacer) with $32 \mathrm{~K}$ memory and single precision arithmetic (6-7 significant digits). To compare computer time requirements it should be noted that a CDC-6600 is about 15-30 times faster than the process computer used. Main points to be discussed in the following examples are:

(i) The necessary value of $\epsilon_{\max }$, where $\epsilon_{\max }$ is the specified relative maximum error of the spatial approximation (relative with respect to the variation range of the variable considered, e.g. $c_{0}, \Delta T_{a d}$ etc.). A sufficient error bound $\epsilon_{\max }$ is defined as a value below which no change of plotted dynamic and steady state profiles can be observed.

(ii) The number of grid points used is compared to the number of grid points in a corresponding equidistant grid. A corresponding equidistant grid consists of space steps with the minimum step size used. Since the computation time is roughly proportional to the number of space steps, the ratio of corresponding equidistant grid points to the number of grid points used determines the saving in computation time.

\section{Example 1. Oscillating homogeneous tubular reactor}

The first example is taken from a paper of Varma and Amundson [11] where it was shown that a diffusion type model for the nonadiabatic homogeneous tubular reactor can exhibit oscillations comparable to limit cycles in the CSTR. In dimensionless form the balance equations and boundary conditions for a first order exothermic reaction are:

Mass balance:

$$
\frac{\partial c}{\partial t}=\frac{\partial^{2} c}{\partial z^{2}}-P e_{m} \frac{\partial c}{\partial z}-\alpha r(c, T) .
$$

Energy balance:

$$
\frac{\partial T}{\partial t}=\frac{\partial^{2} T}{\partial z^{2}}-P e_{h} \frac{\partial T}{\partial z}+\beta \alpha r(c, T)-\gamma\left(T-T_{a}\right)
$$

Reaction rate:

$$
\alpha r(c, T)=c \alpha_{0} \exp (-\delta / T)
$$

Boundary conditions:

$$
\begin{aligned}
& \left.\frac{\partial c}{\partial z}\right|_{z=0}=P e_{m}(c(z=0)-1) ;\left.\quad \frac{\partial c}{\partial z}\right|_{z=1}=0 \\
& \left.\frac{\partial T}{\partial z}\right|_{z=0}=P e_{h}(T(z=0)-1) ;\left.\quad \frac{\partial T}{\partial z}\right|_{i=1}=0 .
\end{aligned}
$$

Parameter values used correspond to Fig. 2.3.1 to 3 in [11] and are given below:

$$
\begin{gathered}
P e_{m}=P e_{h}=5 ; \quad \beta=0.5 ; \quad \delta=25 ; \\
\gamma=12.5 ; \quad \alpha=6.2 \times 10^{10} .
\end{gathered}
$$

As can be seen in Fig. 8, temperature and concentration profiles are relatively smooth and a small number of evenly distributed grid points should be sufficient. This holds true for the steady state where 9 grid points are used if $\epsilon_{\max }=0,01$ is specified as error bound. The solution obtained is in good agreement with the more accurate calculations. However, it turns out that the numerical solution of the dynamics strongly depends upon the spatial accuracy. The relative error limit has to be chosen as low as $\epsilon_{\max }=2 \times 10^{-4}$ to obtain satisfactory results. With $\epsilon_{\max }=10^{-3}$ the oscillations calculated are much smaller, with $\epsilon_{\max }=0,01$ the numerical solution shows decaying oscillations and approaches steady state. Limit cycles for $\epsilon_{\max }=10^{-4}$ (accurate solution) and $\epsilon_{\max }=10^{-3}$ are given in Fig. 9.

It should be stated and will be shown in subsequent examples that the requirement of such high accuracy is not typical for diffusion type model calculations but

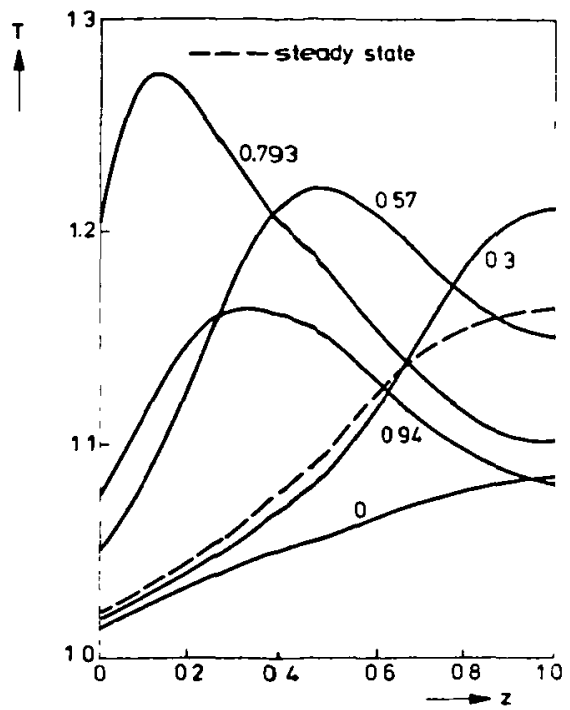

(a)

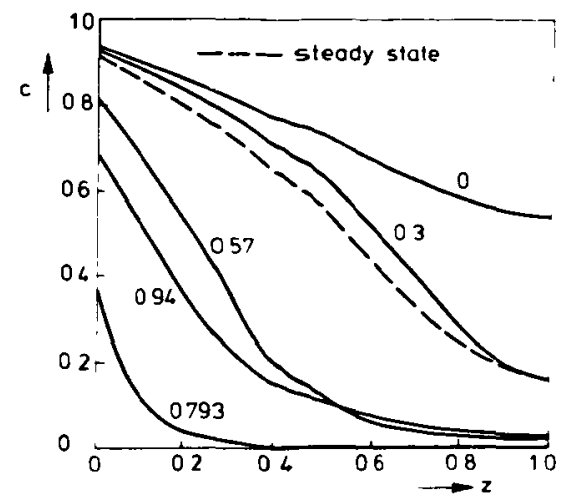

(b)

Fig. 8. Example 1: Dimensionless concentration and temperature profiles in an oscillating tubular reactor $\left(\epsilon_{\max }=10^{-4}\right)$. 


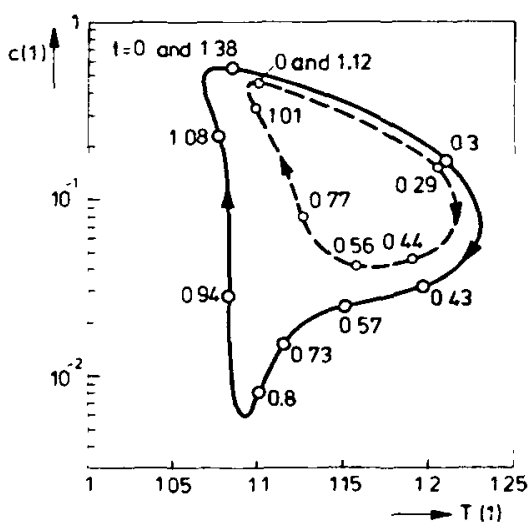

Fig. 9. Limit cycle (exit concentration vs exit temperature) of example I. Solid line: $\epsilon_{\max }=10^{-4}$, broken line: $\epsilon_{\max }=10^{-3}$.

results from the peculiar problem where the instability is brought about by the competing influence of the diffusion of heat and mass. Hence the dynamics are strongly affected by minor errors in the concentration and temperature profiles.

For the accurate solution $\left(\epsilon_{\max }=10^{-4}\right)$, between 24 (at $t=0$ ) and 42 (at $t=0.57$ ) grid points were required and the computation time for one limit cycle was $5 \mathrm{~min}$. Compared to 101 equidistant grid points (as used in the calculations of Varma and Amundson [11]) a factor 3 in computer time can be saved.

\section{Example 2. Steep axial gradients in the fixed bed reactor}

The second example is adopted from a paper of van Doesburg and de Jong[12]. They showed that the hydrogenation of $\mathrm{CO}$ and $\mathrm{CO}_{2}$ in an adiabatic fixed bed reactor with high flow rates is well described by a simple plug flow type model. However, if the flow rate is reduced, axial heat conduction and heat losses to the surroundings will play a more significant role. This case, which will frequently be encountered in laboratory-type studies, is considered here.

For the $\mathrm{CO}_{2}$-hydrogenation with hydrogen in high excess the following model applies:

Mass balance $\mathrm{CO}_{2}$ :

$$
\frac{\partial x}{\partial t}=D_{\text {eff }} \frac{\partial^{2} x}{\partial z^{2}}-v \frac{\partial x}{\partial z}-r(x, T) \approx 0 .
$$

\section{Energy balance:}

$$
\frac{\partial T}{\partial t}=\frac{\lambda_{\text {eff }}}{\overline{\rho c_{p}}} \frac{\partial^{2} T}{\partial z^{2}}-\epsilon v \frac{\rho_{c} c_{p s}}{\overline{\rho c_{p}}} \frac{\partial T}{\partial z}+\frac{2 k w}{R_{T} \rho c_{p}}\left(T_{s}-T\right)+0.0165 r(x, T) .
$$

Reaction rate:

$$
r(x, T)=0.971 \times 10^{13} \frac{x \exp (-12733 / T)}{1+12.7 x} .
$$

Boundary conditions:

$$
\begin{array}{r}
\left.\frac{\partial x}{\partial t}\right|_{z=0}=\frac{v}{D_{\text {eff }}}\left(x(z=0)-x_{0}\right) ;\left.\quad \frac{\partial x}{\partial z}\right|_{z=1}=0 \\
\left.\frac{\partial T}{\partial t}\right|_{z=0}=\frac{\epsilon v \rho_{G} c_{P G}}{\lambda_{\text {eff }}}\left(\mathrm{T}(\mathrm{z}=0)-\mathrm{T}_{0}\right) ;\left.\quad \frac{\partial \mathrm{T}}{\partial z}\right|_{z=1}=0 .
\end{array}
$$

Parameter values are given in Table 2.

The diffusion term in the mass balance is of negligible importance. However, it is not advisable to skip it entirely since the diffusion term has a smoothing influence upon the numerical solution of the mass balance equation in regions where no or minor reaction takes place. It is a general result that with zero diffusion the central difference approximation for the spatial derivative is unstable, resulting in "rough" solution profiles. In our case the space step control algorithm tends to place additional grid points within the noisy region which makes things even worse. The application of the method therefore should be limited to Peclet numbers below 1000 (which in most cases is essentially the same as plug flow).

Figures 10 and 12 show temperature transients of the model due to step changes in feed temperature. The mass balance for $\mathrm{CO}_{2}$ was considered in quasi steady state. Calculations with both $\epsilon_{\max }=0.01$ and 0.003 were performed and showed that the larger value was generally

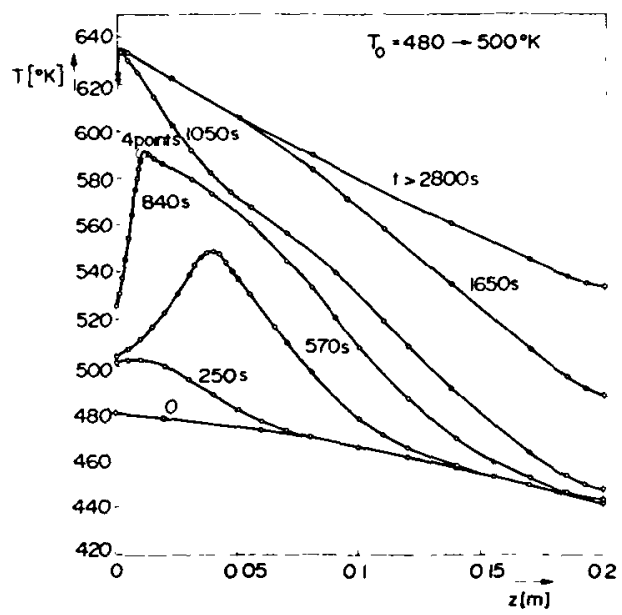

Fig. 10. Example 2: Development of the temperature profile and grid point distribution during reactor start-up $\left(\epsilon_{\max }=0.003\right)$. The circles mark the current position of the grid points.

Table 2. Parameter values for Example 2

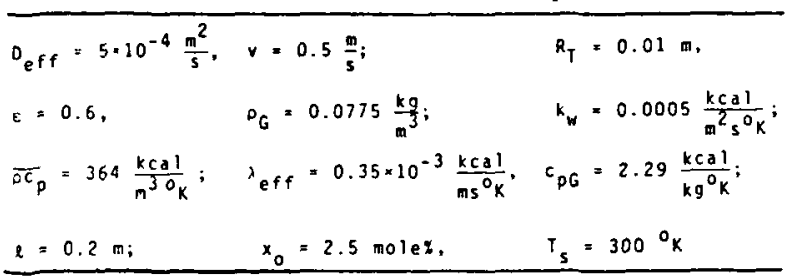


sufficient. As the temperature profile develops, the space grid changes as can be seen in Fig. 10. In the steady state at $T_{0}=500^{\circ} \mathrm{K}$ half of the total number of 18 grid points are concentrated within the first percent of the reactor length. A section of the resulting steady state profile is shown in Fig. 11. It can be seen that the distance between subsequent grid points can differ by a factor of 10 and more.

The method shows its prime advantages in the calculation of creeping profiles (Fig. 12). To demonstrate the influence of the error bound, the grid point distribution in Fig. 12 at $t=2250 \mathrm{~s}$ is taken from a run with $\epsilon_{\max }=0.003$, and the distribution at $t=3250 \mathrm{~s}$ from a run with $\epsilon_{\max }=0.01$. It should be noted that only $25 \%$ more grid points are required in order to triple the accuracy.

A maximum of 22 grid points for ignition and 32 points for extinction were used for $\epsilon_{\max }=\mathbf{0 . 0 1}$. If the error bound was specified as $\epsilon_{\max }=0.003$ the corresponding values

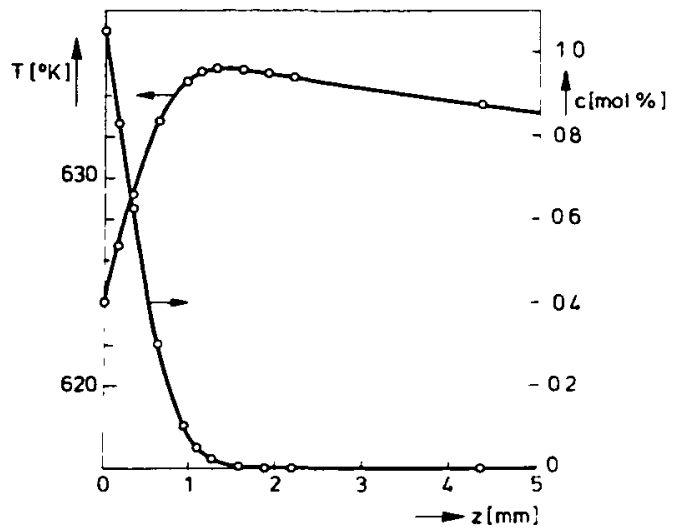

Fig. 11. Section of the steady state profiles and position of grid points (circles) at $T_{0}=500^{\circ} \mathrm{K}, \epsilon_{\max }=0.003$.

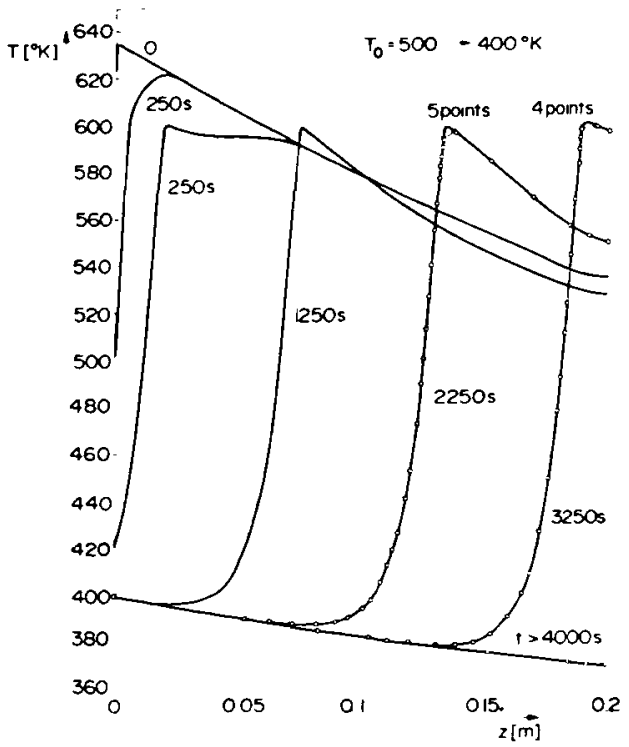

Fig. 12. Blow-out of the reaction. Grid point distribution (circles) at $t=2250 \mathrm{~s}$ obtained with $\epsilon_{\max }=0.003$, at $t=3250 \mathrm{~s}$ with $\epsilon_{\max }=0.01$. were 32 and 43 . Computation times required were $2.5 \mathrm{~min}$ for start up and $11 \mathrm{~min}$ for blow out with $\epsilon_{\max }=0.01$, and $4 \mathrm{~min}$ and $25 \mathrm{~min}$ respectively for $\epsilon_{\max }=0.003$. To obtain the same accuracy as in the calculations with $\epsilon_{\max }=0.01$, an equidistant grid with 650 points for ignition and 300 points during blow out would have been necessary. Hence a factor between 10 and 30 in computation time has been saved. For the lower error bound the savings are even higher.

Example 3. Fixed bed reactor with inert portions experiencing catalyst decay

The third example is the more complex problem of a reaction under the influence of catalyst deactivation in a fixed bed reactor consisting of inert and active portions. This problem has been treated in detail by Weng et al. [13]. At issue was the irreversible poisoning of a nickel catalyst by thiopene in the hydrogenation of benzene. It turned out that a diffusion type model as in Example 2 was able to describe the experimental results satisfactory. The model consists of the following four balance equations:

Mass balance reactant (benzene):

$$
\frac{\partial c_{B}}{\partial t}=D_{B} \frac{\partial^{2} c_{B}}{\partial z^{2}}-v \frac{\partial c_{B}}{\partial z}+\frac{\rho_{c}}{\epsilon} r_{B}\left(\theta_{A}, c_{B}, T\right) \approx 0 .
$$

\section{Mass balance poison (thiophene):}

$$
\frac{\partial c_{T}}{\partial t}=D_{T} \frac{\partial^{2} c_{T}}{\partial z^{2}}-v \frac{\partial c_{T}}{\partial z}+\frac{\rho_{\mathrm{C}}}{\epsilon} r_{T}\left(\theta_{A}, c_{T}, T\right) \approx 0
$$

Energy balance:

$$
\begin{gathered}
\frac{\partial T}{\partial t}=\frac{\lambda_{\text {eft }}}{\rho c_{p}} \frac{\partial^{2} T}{\partial z^{2}}-\epsilon v \frac{\rho_{G} c_{p G}}{\rho c_{p}} \frac{\partial T}{\partial z}+\frac{2 \alpha}{R T \rho c_{p}}\left(T_{n}-T\right) \\
-\frac{\left(-\Delta H_{R}\right)}{\overline{\rho c_{p}}} \rho_{c} r_{B}\left(\theta_{A}, c_{B}, T\right) .
\end{gathered}
$$

Mass balance active sites:

$$
\frac{\partial \theta_{A}}{\partial t}=r_{d}\left(\theta_{A}, c_{T}, T\right)
$$

(Hydrogen was in great excess, so the mass balance for hydrogen was not required.) Reaction rate expressions and values of the constants are given in [13]. The reactor consisted of two portions of inert material at both ends (extending from $z=0$ to $z_{1}$ and from $z=z_{2}$ to $l$ ) and a section of active catalyst in the middle (between $z=z_{1}$ and $z_{2}$ ). Hence the mass balance equations needed only to be solved for the active section of the bed, and since eqns (27) and (28) were in quasi steady state, the simplified Wehner-Wilhelm boundary conditions (which coincide with the Dankwerts conditions) applied:

$$
\left.\frac{\partial c}{\partial z}\right|_{z=z_{1}}=\frac{v}{D}\left(c\left(z=z_{1}\right)-c_{0}\right) ;\left.\quad \frac{\partial c}{\partial z}\right|_{:=z_{2}}=0
$$

The energy balance however had to be solved over the entire length of the reactor. Since it was assumed that heat 
conductivity in active and inert portions was the same, only boundary conditions at the reactor entrance and exit have to be considered:

$$
\left.\frac{\partial T}{\partial z}\right|_{z=0}=\frac{\epsilon v \rho_{E} c_{p G}}{\lambda_{\text {eff }}}\left(T(z=0)-T_{0}\right) ;\left.\quad \frac{\partial T}{\partial z}\right|_{z=1}=0
$$

In the inert portions the reaction rate term in eqn (29) is equal to zero which means that the source term of the energy balance jumps at the boundaries from inert to active sections of the bed. This is shown in Fig. 13. Four fixed grid points ( $L 10, L 10+1$ and $L 20, L 20+1)$ have to be used to specify the boundaries $z_{1}, z_{2}$ between inert and active portions. Only in this way it can be ensured that an element belongs completely to the inert or completely to the active portion. Source term corrections can of course only be made with values from either the inert or the active portions.

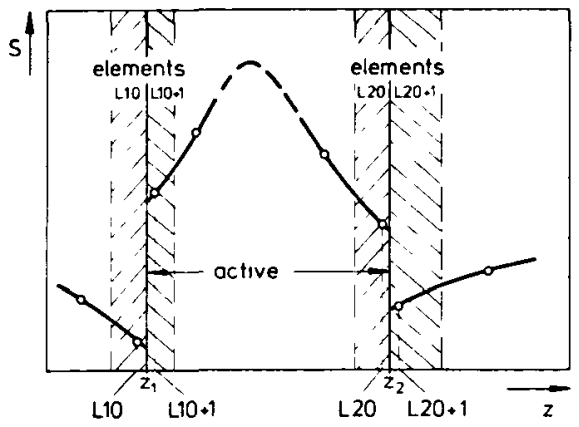

Fig. 13. Source term of the energy balance at the boundary between inert and active portions of the bed and location of grid points and elements.

Results of the calculation are given in Fig. 14. Again an error bound of $\epsilon_{\max }=\mathbf{0 . 0 1}$ proved sufficient. About 30 grid points were used, one third of which were located in both inert portions. The minimum distance between adjacent grid points within the active portion was $0.15 \mathrm{~mm}$ which amounts to 200 equidistant grid points for the whole reactor. Hence a factor 6 in computation time was saved.

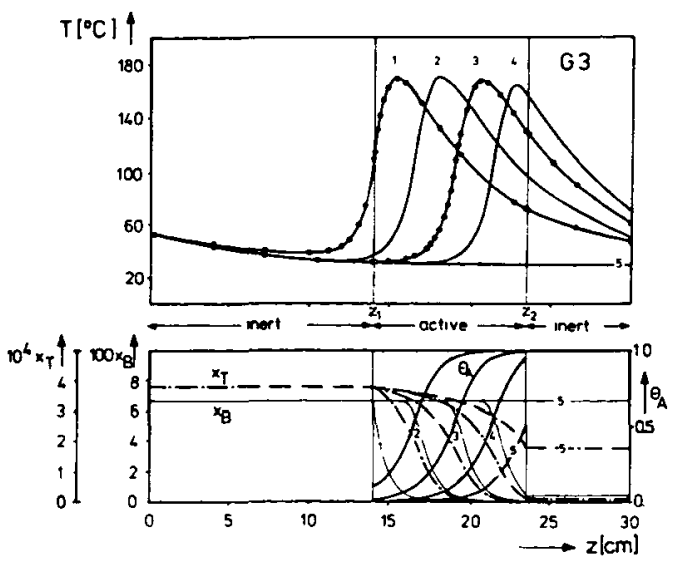

Fig. 14. Profiles of reaction variables in Example 3. Time marks: 1-steady state with poison free feed; $2-30 \mathrm{~min} ; 3-60 \mathrm{~min}$; $4-90 \mathrm{~min} ; 5-120 \mathrm{~min}$ after onset of poisoning.

\section{CONCLUSIONS}

A solution method for diffusion type models based upon a non-equidistant spacing scheme has been derived. It is applicable to diffusion problems and to diffusionconvection problems with $P e$-numbers below 1000 and has the following main advantages:

(1) Accuracy bounds for the spatial accuracy can be specified and the solution will always be kept within this specified range by automatic insertion or elimination of space grid points. For most cases an upper relative error bound $\epsilon_{\max }$ between 0.005 and 0.01 and a lower limit $\epsilon_{\min }=0.1 \epsilon_{\max }$ give satisfactory results.

(2) Due to the automatic elimination of grid points in regions with flat solution profiles a considerable reduction in the total number of axial grid points required can be achieved. This results in high savings of computer time and storage space. The method shows its main advantages if very steep solution profiles occur. In these cases a factor 10 and more in computer time can be saved as compared to computations in an equidistant grid. High computation speed and moderate storage requirements make the method well suited for use on process computers.

(3) The finite difference approximation with nonequidistant space steps converts partial differential equations into a low order system of ordinary differential equations (eqn 10). Thus it is possible to consider problems of stability and control via the (linearized) system of ordinary differential equations, which has considerable advantages over other methods in case of steep solution profiles. This problem will be discussed in a separate contribution.

Acknowledgements-This work was supported by the Deutsche Forschungsgemeinschaft and by the National Science Foundation, GK 17200.

\section{NOTATION}

$A$ reactor cross section

$A L$ constant of finite difference approximation of balance equations (eqns 13 and 14, and Table 1)

B constant of source term correction (eqn 20 , Table 1)

c concentration

C constant of spatial derivative approximation (eqns 4-9)

$c_{p}$ specific heat

$D_{\text {ef }}$ effective diffusity

DA constant of finite difference approximation of balance equations (eqns 13 and 14)

$D D$ constant of finite difference approximation of balance equations (eqn 13)

$D E$ constant of spatial derivative approximation at the boundaries (eqns 6 and 9 , and Table 1)

$D Z M, D Z P$ spatial differences between grid points $\operatorname{DZM}(L)=D Z P(L-1)=z_{L}-z_{L-1}$

$E$ error of spatial approximation (eqn 15)

$f$ function of $y$ 
$H M, H P$ constants for the calculation of half-values Indices

YHM, YHP (eqn 16, Table 1)

$k_{w}$ heat transfer coefficient

1 reactor length

$L$ space grid point number (index)

LM total number of space grid points; last grid point (at $z=l)$

$r$ reaction rate

$R_{T}$ tube radius

$S$ source term

$\bar{S}$ mean value of $S$ in interval (eqn 20)

$t$ time

$T$ temperature

$T_{S}$ ambient temperature

$v$ interstitial mean velocity

$x$ mole fraction

$y$ dependent variable

yo feed value of dependent variable

$Y H M, Y H P$ half values of dependent variables in middle of interval (eqn 16, Fig. 5)

$z$ spatial coordinate

$\Delta t$ time step

$\left(-\Delta H_{R}\right)$ reaction enthalpy

$\Delta T_{a d}$ adiabatic temperature rise

Greek symbols

$\epsilon$ void fraction

$\epsilon_{\mathrm{mm}}$ minimum value of the relative spatial error

$\epsilon_{\max } \operatorname{maximum}$ value of the relative spatial error

$\theta_{A}$ active site density

$\rho$ density $c$ catalyst phase

$G$ gas phase

$k$ time index

$L$ space grid point index

$p$ iteration index

0 feed value

\section{REFERENCES}

[1] Crank J. and Nicolson P., Proc. Cambridge Philos. Soc. 1947 4350.

[2] Lee E. S., Quasilinearization and Invariant Imbedding. Academic Press, New York 1968.

[3] Villadsen J. V. and Stewart W. E., Chem. Engng Sci. 196722 1483.

[4] Finlayson B. A., The Method of Weighted Residuals and the Variational Principle. Academic Press, New York 1972.

[5] Lübeck B., Chem. Ing. Techn. 19714397.

[6] Lübeck B., Chem. Engng Sci. 1974291320.

[7] Lübeck B., Dissertation Universität Stuttgart 1974.

[8] Lübeck B., Chem. Engng J. 1974729.

[9] Carey G. F. and Finlayson B. A., Chem. Engng Sci. 1975 30587.

[10]. Pearson C. J., Math. Phys. 196847351.

[11] Varma A. and Amundson N. R., Can. J. Chem. Engng 1973 51459.

[12] Van Doesburg H. and de Jong W. A., Int. Symp. Chem. React. Engng Evanston (1974), Advances in Chem. Series. $133,489$.

[13] Weng H. S., Eigenberger G. and Butt J. B., Chem. Engng Sci. 1975301341.

[14] Osher S., SIAM J. Numer. Anal. 19707199.

[15] Ciment M., Math. of Comput. 197125219.

[16] Browning G., Kreiss H. O. and Oliger J., Math. of Comput. 19732729.

[17] Venit S., Math. of Comput. 197327745.

[18] Ciment M. and Sweet R. A., J. Comput. Phys. 197312513. 